\title{
Accommodated Emoji Usage: Influence of Hierarchy on the Adaption of Pictogram Usage in Instant Messaging
}

\section{Tobias Kroll}

Department of Computer Science and Applied Cognitive Science

University of Duisburg-Essen

Duisburg, Germany

Email: tobias.kroll@uni-due.de

\section{Lea-Marie Braun}

Department of Computer Science and Applied Cognitive Science

University of Duisburg-Essen

Duisburg, Germany

Email: lea-marie.braun@uni-due.de

\section{Stefan Stieglitz}

Department of Computer Science and Applied Cognitive Science University of Duisburg-Essen

Duisburg, Germany

Email: stefan.stieglitz@uni-due.de

\begin{abstract}
Communication Accommodation Theory predicts to what extent individuals accommodate their verbal and nonverbal behaviour by converging it towards their conversation partner or diverging it away from them to gain social approval and to decrease social distance. Especially individuals in lower hierarchy positions accommodate their communication behaviour towards individuals in higher hierarchy positions. Nowadays, computer- and smartphone-mediated communication are common ways to communicate, for example via instant messaging. However, instant messenger lack in transporting nonverbal cues. To fill this gap, emoji are used increasingly. A study was conducted to examine how individuals in lower hierarchy positions converge their emoji usage towards individuals in higher hierarchy position. The results support the assumption that the higher hierarchy is perceived, the more emoji accommodation is shown.
\end{abstract}

Keywords Communication Accommodation Theory, Convergence, Emoji, Social Hierarchy, Mobile Communication 


\section{Introduction}

In recent years, smartphones have become disruptive high-tech devices which most people use on a daily basis (Lee et al. 2014). Functions provided in smartphones help to manage everyday tasks and offer ubiquitous communication channels (Park et al. 2014). Besides traditional calls and steady mail access, instant messaging (e.g. iMessage, WhatsApp) has become popular with the rise of Internet-capable devices (Church and de Oliveira 2013; Stieglitz et al. 2014). This new role of instant messaging also in the business communication (Muir et al. 2017) raises questions about its influence in organisations, e.g. by with regard to secure communication channels, technostress, work life balance, but also on the communication culture between employees or on hierarchical structures. Enterprise social networks (sometimes including instant messaging) have already related to less hierarchical communication and reduced social distances (Riemer et al. 2015). New instant messaging services enable a plethora of research areas within the field of information systems and computer-mediated communication, especially when integrating different perspectives.

From prior research, we learned that in face-to-face (FtF) communication, people show the tendency to (unconsciously) adopt their interlocutors' language as phrases and accents as well as the nonverbal behaviour as facial expressions and gestures (Giles and Ogay 2007). This behaviour is called accommodation and has been studied and explained by the Communication Accommodation Theory (CAT) (Giles and Ogay 2007, p. 293). CAT addresses accommodations individuals make either towards their conversation partners or away from them. Usually, accommodation aims to increase the social acceptance and to smooth interaction. In particular, it has been observed that people in a lower hierarchical position adopt the language of interlocutors in higher hierarchical positions (Giles et al. 1991).

While facial expressions cannot be accommodated in instant messaging, phrases and word orders can be equalized (Muir et al. 2017). Due to the lack of possibilities to reveal emotions or gestures, emoji have been developed out of simple smiley representations (Chen et al. 2017). With emoji, it is possible to add a certain expression or emotion to messages and to enrich and potentially improve them in terms of unambiguous comprehension (Li et al. 2018). Emoji usage may support reducing the distance between individuals in a lower hierarchical position and those in a higher hierarchical position which leads to possibly being able to avoid communication problems based on hierarchy (Skovholt et al. 2014). Further, knowledge about emojis and their impact on conversations' effectiveness is especially relevant for organisations as these replace more and more FtF meetings by virtual communication and collaboration (Gilson et al. 2015).

Although emojis are seen as helpful tool to substitute nonverbal cues in computer-mediated communication and instant messaging (Chen et al. 2017; Walther and D'Addario 2001), to the best of our knowledge their usage has not yet been studied in relation to hierarchy and organisational communication. This study's aim is to examine the emoji accommodation behaviour in instant messaging. Our study builds upon the Communication Accommodation Theory (CAT) which has received only little attention in information systems, yet (e.g. Ludwig et al. 2014). The guiding research question is: What is the connection between the usage of emoji and the perceived hierarchy in instant messaging communication?

With the focus on instant messaging and hierarchy, we originally use the Communication Accommodation Theory to empirically analyse and explain different emoji usage behaviours. This study contributes to the understanding of today's communication and provides insights about the effect of emoji usage on the perceived hierarchy as well as the effect of perceived hierarchy on emoji accommodation behaviour.

\section{Background}

\subsection{Computer-mediated Communication in Organisations}

The development and prevalence of devices like smartphones in the last decade has constituted the replacement of traditional Face-to-Face (FtF) communication with computer-mediated communication (Waldeck et al. 2012). Older mobile devices with a small and monochrome display as well as limited and expensive Internet access have turned into high-performing allrounder smartphones (Park et al. 2014) which are used by most people daily (Lee et al. 2014). They offer many advantages by means of constantly developing functions helping to manage daily routines - both private and business (Meske et al. 2018). Mobility is an important factor in this context because it gives users the chance to communicate at any time and location (Church and de Oliveira 2013; Stieglitz et al. 2014). Likewise, 
instant messaging has been increasingly adopted from organizations (Wang and Gallivan 2009) and has changed the way employees collaborate and communicate (Cheung et al. 2007). Organisations evaluated instant messaging to be quicker and more comfortable than email or phone calls (Muir et al., 2017) because of quasi real-time text messages and ancillary presence signals (Wang and Gallivan 2009) which support collaboration and communication processes (Cheung et al. 2007).

Besides communication in teams or between employees in general, communication between superiors and subordinates is crucial for effective organisational operations (Myers 2016). Many organisations have the structure of formal or social hierarchies meaning the ranking of individuals in a social group; some members are superior while others are subordinate. Ranking is about the positions someone has in a hierarchy based on a valued social dimension; a higher rank often goes hand in hand with higher social power (Koski et al. 2015). The different positions within an organisational hierarchy provide knowledge and clarity about how to act in certain situations and, hence, they support individuals' desire to behave appropriately (Halevy et al. 2011). Clear roles and responsibilities reflected in legitimate hierarchies are helpful for a coordinated and productive working environment.

Research found that superiors' communication style has an influence on the communication quality and on relationship building (Myers 2016). Strong relationships between superiors and subordinates are important since they affect the way subordinates feel connected and integrated in the whole organisation. This, in turn, increases performance and reduces the chance of termination (Bisel et al. 2012). Communication is more effective when superiors communicate with openness, sensitivity to others, good listening skills and persuasiveness (Myers 2016). Further, communication is shaped by nonverbal behaviour. They are able to include nonverbal cues in their message to signal liking or positive feedback for their subordinates (Richmond and McCroskey 2000). Furthermore, superiors that exhibit high nonverbal immediacy are perceived as more credible and more interpersonally attractive than superiors that use nonverbal immediacy rarely (Richmond and McCroskey 2000). Perceived credibility and attractiveness affect subordinates' motivation and job satisfaction. Moreover, superiors that engage in nonverbal behaviour are evaluated more positively and subordinates appreciate this kind of communication. When both superiors and subordinates add nonverbal behaviour to their messages, they have a stronger relationship.

However, the increased usage of instant messaging in organisations has also been suspected to hinder the quality of a superior-subordinate communication and to influence the relationship negatively (Kirkman et al. 2002; Quan-Haase et al. 2005). Especially the instant messaging's lack of nonverbal cues that provides awareness has been criticised (Church and de Oliveira 2013). Still, developers have tried to fill the gap by adding new options like symbols to confirm delivery or reading, but still, nonverbal cues as gestures, facial expressions and body language could hardly be conveyed via instant messaging (Park et al. 2014). Consequently, the lack of nonverbal cues may have a negative impact on both sender and receiver. Senders cannot easily convey emotions with a message nor consider their interlocutor's mood. The receivers' challenge is again to interpret a message's meaning with the absence of nonverbal cues (Mirbabaie et al. 2017).

\subsection{Emoji as Nonverbal Cues}

Due to the missing possibility to textually convey certain information, users started to add characters to text messages that form emoticons - e.g. ;-) - and aim at conveying information like a sentiment or intended irony (Mirbabaie et al. 2017; Riordan 2017a). However, more complex objects and emotions cannot be represented by emoticons since the typographic symbols are limited (Lu et al. 2016). To address this limitation, a Japanese graphic designer transformed emoticons into Unicode characters named emoji (a combination of the Japanese words for "picture" and "character") in the year 2009 (Riordan 2017b; Wijeratne et al. 2017). Today, emoji show a big variety of signs and symbols like food, flags, animals and clothing (Chen et al. 2017). The most popular emoji, though, are the face-related ones that express a certain emotion (Chen et al. 2017). Emoji got a lot of attention and became increasingly popular - also indicated by the Oxford Dictionaries Word of the Year 2015, "Face with Tears of Joy". Examining their popularity, a first explanation might be the compactness of emoji. People do not have to explain each of their emotions but can add one emoji to their message to convey a feeling that would be hidden using text only (Lu et al. 2016). For example, "I got my test results " conveys another message than "I got my test results ${ }^{-0}$ " (Pohl et al. 2017). Tandyonomanu and Tsuroyya (2018) found that emoji can reduce misunderstandings and give individuals the chance to show their emotions. Further, they can be seen as a way to replace certain nonverbal cues known from FtF communication in a computer-mediated communication (Chen et al. 2017; Walther and D'Addario 2001). In this context, it has been found that not all individuals use emoji the same way nor do they interpret them equally 
(Tigwell and Flatla 2016). Older people use less emoji than younger ones (An et al. 2018), females tend to use more emoji than males do (Chen et al. 2017) and also, personality influences emoji usage (Li et al. 2018).

Elder (2018) argues that receiving messages with emoji have a positive influence on how the receiver perceives, interprets and replies. Receivers tend to be more altruistic, honest and generous in case they received a message that includes face emoji. Those emotions, again, have a positive effect on the answer and can support relationship building as well as friendship strengthening (Elder 2018). Perceiving facial expressions leads to interpersonal social mechanisms like emotional contagion and supports relationship building (Lohmann et al. 2017). However, emoji can also have a dark side because they show more the expressions and emotions individuals want their interlocutors to perceive than the emotions they really feel (Tandyonomanu and Tsuroyya 2018). Emoji have the ability to hide real feelings. Furthermore, they have been evaluated as inappropriate and harmful for the sender's credibility in business contexts (Glikson et al. 2017).

\subsection{Communication Accommodation Theory}

The Communication Accommodation Theory (CAT) explains an individual's communicative adjustments towards or away from others to create, maintain or decrease social distance (Giles and Ogay 2007). Such constantly changing communication behaviour is called accommodation. CAT is based on the main assumption that individuals adjust themselves depending on their conversation partner (Dragojevic et al. 2016). This behaviour is pervasive and part of a successful interaction. Accommodations are made immediately and mostly unconsciously. It can be separated in three different strategies: convergence, divergence and maintenance (Dragojevic et al. 2016; Giles and Ogay 2007). Convergence is about adjusting one's communication behaviour in a way to be more like the conversation partner. The goal is to smooth interaction and to decrease social distance. Contrary with divergence, individuals adjust their communication behaviours to be more dissimilar to their conversation partners.

Individuals do not only adjust their verbal expressions but also their nonverbal behaviour. CAT has contributed to the explanation of how and why communication is accommodated and its consequences (Giles and Ogay 2007). Since nonverbal cues cannot be observed in textual computer-mediated communication, researchers investigated whether verbal communication and potentially more dimensions are accommodated. Bunz and Campbell (2004) found that structural politeness phrases are accommodated during email communication. When participants received a friendly written email with structural and verbal politeness phrases (e.g. Dear Mrs. ... Kind regards), they answered with a significantly politer email than without such phrases. In the context of online communities, it has been found that users tend to converge their linguistic style (e.g. the usage of functional words) on a higher degree when they feel stronger connected to their community (Ludwig et al. 2014).

In addition to verbal cues, nonverbal cues like response time and message length are accommodated (Riordan et al. 2013). Scissors et al. (2009) found that individuals also accommodate on three different levels: content (semantic), structural (tenses or syntactic) and stylistic (surface) level. Creating similarity on a content level may be realized by having the same opinion about a topic. At the structural level, similarity can be increased by using the same tense or repeating phrases. To create similarity on the stylistic level, individuals copy certain words (e.g. LOL) or punctuations (e.g. !!!).

\subsection{Accommodation and Hierarchy}

Considering the interplay of two individuals communicating, CAT describes a symmetrical and asymmetrical accommodation (Dragojevic et al. 2016). In particular, hierarchical imbalance facilitates asymmetrical convergence (Giles et al., 1991), i.e. individuals in lower hierarchical position converge their communication behaviour more to individuals in higher hierarchical position than the opposite direction (Jones, Gallois, Callan, \& Barker, 1999). CAT explains that individuals in lower hierarchical position are motivated to accommodate their communication behaviour to gain social approval and to minimize social distance (Giles et al. 1991; Muir, Joinson, and Cotterill 2016).

Some studies concluded that the instant messaging between superiors and subordinates can increase social distance and complicate the relationships between them because of missing nonverbal and social cues and reduced richness (Kirkman et al. 2002; Quan-Haase et al. 2005). A harmed relationship can hinder effectiveness and decrease performance (Myers 2016). However, confirming results from FtF communication, Muir et al. (2017) found instant messaging users in lower hierarchical positions to be more likely to accommodate their linguistic style to those in a higher hierarchical positions than the 
opposite direction. Furthermore, this upward accommodation leads to positive interpersonal impressions and task attractiveness (Muir et al. 2017).

Summarising and deriving our hypotheses, imbalanced hierarchical situations have been shown to affect the degree of accommodation behaviour (Muir et al., 2017). In particular, people show convergence of e.g. message length, response time, politeness phrases and linguistic style (Bunz and Campbell 2004; Riordan et al. 2013; Scissors et al. 2009). In FtF communication, verbal and nonverbal cues are expected to play a crucial role and were both found to be accommodated. Emoji are a rather new way to substitute nonverbal cues in computer-mediated communication but have not yet been studied in this light before. Also, emoji have been discussed as inappropriate for business communication and imbalanced hierarchical positions (Glikson et al. 2017). However, it is yet to be studied how emoji usage affects the perceived hierarchical position as well as how the perception of hierarchy influences the emoji accommodation behaviour. Considering and synthetizing previous findings, we hypothesize:

H1: Individuals in low perceived hierarchical positions will accommodate their instant messaging emoji usage more than individuals in high hierarchical positions.

H2: The higher individuals perceive their interlocutors' hierarchy position, the more they show emoji accommodation in instant messaging.

H3: Instant messaging with emoji leads to a lower perceived hierarchical distance than instant messaging without emoji.

\section{Method}

The proposed hypotheses were investigated quantitatively in an online experiment. The basic study design was based on Muir et al. (2017) and included four different instant messaging chat records in a usual messenger look. The interlocutor's messages were predefined. Such messages differed in two dimensions: (1) formal versus informal, and (2) emoji usage versus no emoji usage. Two variants are shown in figure 1. In each chat record, three placeholders between the interlocutor's messages had to be filled. For each placeholder, a textual answer without any emoji was given. The participants' only task was to decide whether to add emoji to the prepared text or not. Thereby, they could choose none, one or more of the fifteen most used emoji. Referring to Jaeger et al. (2018), the emoji were picked out based on the website Emoji tracker that analyses Twitter posts and depicts emoji frequencies. Only facial and

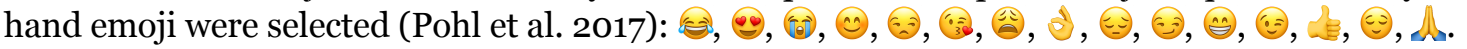

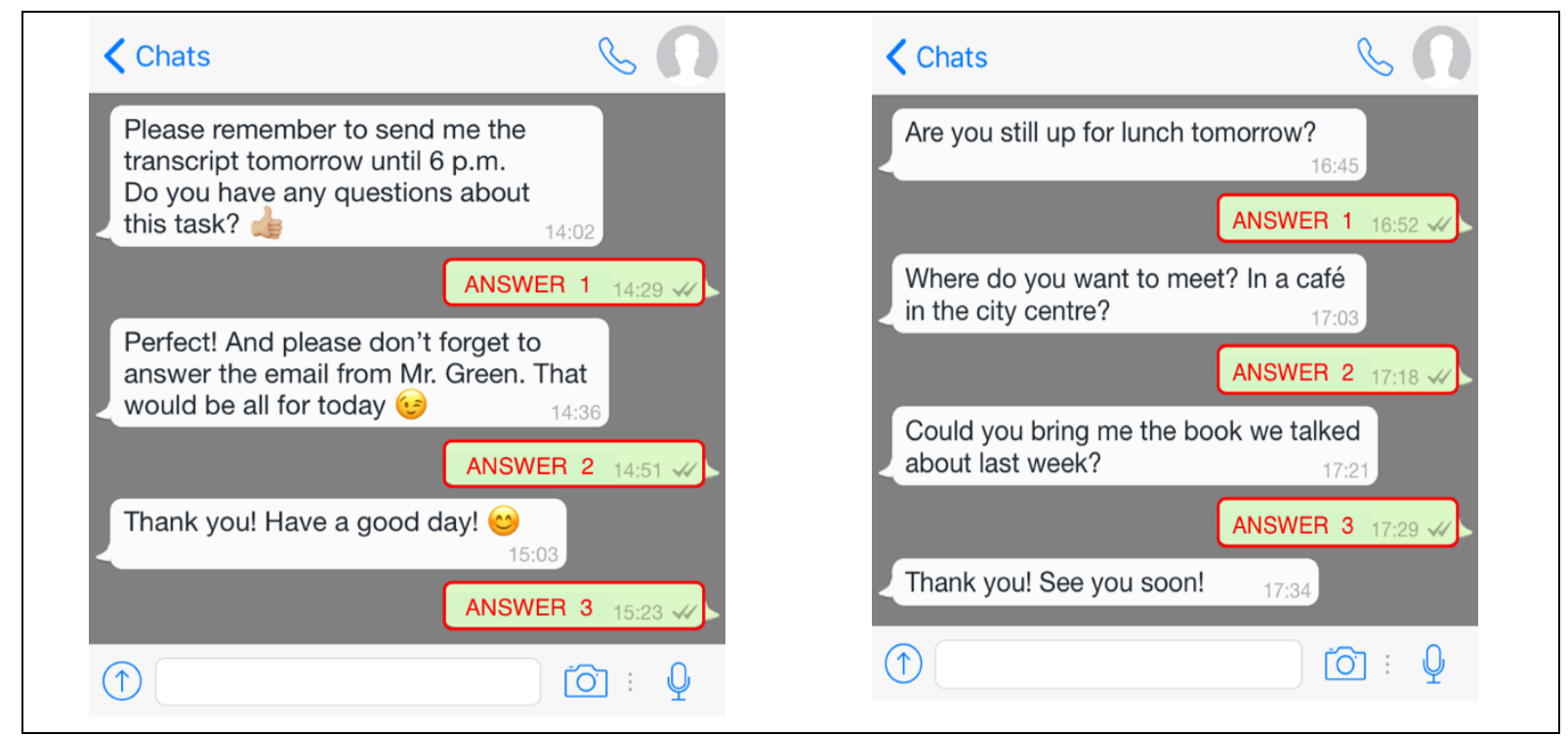

Figure 1: Formal chat with emoji (left), informal chat without emoji (right)

After each chat record, the participants were asked to indicate the interlocutor's perceived hierarchical position based on a 5-point Likert scale from "Lower hierarchical position" to "Higher hierarchical position". By design, we expected the formal chat to be perceived as a higher hierarchical position (e.g. chat with the boss) and the informal chat record as a conversation with an individual in about the same hierarchical position as (e.g. friend). We did not include a conversation with a lower hierarchical position 
because we believed that most participants would perceive it as artificial. We have captured the perceived hierarchy to divide the study participants more precisely into groups (i.e. interlocutor in higher position versus interlocutor in equal or lower position). We assumed the perception of a same chat to be perceived hierarchically to varying degrees. Participants also had to fill in a questionnaire about their demographics like age, gender and education. Additionally, they were asked for their emoji preference by answering how often they use emoji in computer-mediated communication with four options (never to always) based on Jaeger et al. (2018).

The actual accommodation was measured with the Zelig Quotient developed by Jones et al. (2014). The Zelig Quotient has been used in many studies which examine accommodation (Muir et al. 2017; Muir, Joinson, and Cotterill 2016; Muir, Joinson, Cotterill, et al. 2016). To the best of our knowledge, no one ever examined emoji accommodation which we operationalised as follows: We based the calculations on the general procedure of the Zelig Quotient that measures linguistic style accommodation by counting how often a certain feature is used. A feature is, for example, a pronoun, an article or a conjunction (Muir, Joinson, Cotterill, et al. 2016). In the context of the usual Zelig Quotient, the content of the feature is not important and only the feature's frequency is relevant. Consequently, we also took the number of used emoji as input disregarding the precise emoji and their meaning. The specific calculations are described in the data preparation section.

The participants were shown all four variations in random order to minimize a familiarization effect. Thereby, we could build a baseline of emoji usage meaning the average usage of emoji per message. Furthermore, they had to fill in one of four randomly selected scales of a personality inventory after each chat record to divert their attention slightly to another task. The emoji selection of all four chat protocols served as basis for the assessment of an emoji usage baseline (see next section).

\section{Results}

\subsection{Sample and Procedure}

The participants of the online survey were either recruited via social media or by face to face recruitment. In total, 158 German-speaking participants took part in the online study of which 56 (35.4\%) were male and 96 (60.6\%) female - six did not specify the gender. The mean age was 29.25 with a range from 18 to 65 and a standard deviation of 11.26. Most of the participants were graduated from school (57.6\%). 31 (19.6\%) participants had a bachelor's degree, $29(18,3 \%)$ had master's degree, Diploma or Magister, three participants already received a $\mathrm{PhD}(1,9 \%)$. Further, $46.8 \%$ were students, $2.5 \%$ were trainees, $36.7 \%$ were employees, $1.9 \%$ were self-employed and $3.8 \%$ were civil servants. Like in other studies, a large part of the participants are students. Although they are usually unexperienced as regards business communication and productivity, they are more familiar with emoji and know situations of different hierarchical positions.

\subsection{Data Preparation}

The statistical analyses were carried out by SPSS 25.0. We excluded participants with too short or too long timings as we expected them to have not answered the questions thoroughly. To measure accommodation, the Zelig Quotient was calculated (Jones et al. 2014): The total number of used emoji for each participant was calculated as well at the number of used emoji for each chat record. A baseline was calculated with the average emoji number per answer (total number / 12 answer). Also, the average for each of the four chat records was calculated (number for chat record / 3 answer). The differences between the baseline and the different chat record means were calculated (i.e. Zelig Quotient). An outlier assessment of the quotients led to the exclusion of six further participants. The final data set included 122 participants.

We let the participants select emojis for each of the four records to calculate the emoji baseline. Though people may also accommodate their behaviour e.g. towards peers, we focused on hierarchy and included the hierarchy variants to assess the baseline for different hierarchical positions. For the accommodation analyses, we eventually used only the first randomly selected chat record of each participant and the respective answers for the perceived hierarchy and the selected emoji. Further preparatory work included a test whether the baseline values represent the participants' general tendency to use emoji. The correlation between baseline value and emoji tendency was calculated $(r=.308, p=.001)$. The correlation coefficient is appropriate because of the subjective self-selection into one of four groups in comparison to the average of actually picked emojis. A visual inspection of the accommodation value histogram and Q-Q-Plot showed a good fit for normal distribution. To validate whether the participants perceived the four different chat records as expected, we also tested how the chat records differ from 
each other in their perceived hierarchy position. Therefore, a Friedman's ANOVA was conducted to compare the four chat records (formal without emoji: $M=4.07, S D=1.38$; formal with emoji: $M=3.89$, $S D=1.29$; informal without emoji: $M=2.90, S D=0.63$; informal with emoji: $M=2.85, S D=0.64$ ). Results revealed a general difference between the four chat records, $\chi^{2}(3)=133.26, p<.001$ demonstrating a sufficient experiment design.

\subsection{Perceived Hierarchy and Emoji}

To test hypothesis 1 , we conducted a t-test for independent samples with perceived hierarchical position as independent grouping variable and emoji accommodation as dependent variable. When a participant perceived the interlocutor to be of a higher hierarchical position (perceived hierarchy position $>3$ ), the participant was in a lower hierarchical position. Equally, when a participant perceived their interlocutor as being in an equal or lower hierarchical position (perceived hierarchy position $<=3$ ), the participant saw himself in an equal or higher hierarchical position. For clarification, we did not used the random stimuli (informal without emoji etc.) as separation criterion.

The groups were almost equal: low hierarchical position $(N=60)$ and high hierarchical position $(N=$ 62). Homogeneity of variances was tested using Levene's Test which showed that equal variances could be assumed, $F(120)=1.92, p=.169$. The results of the t-test showed that on average participants in a low hierarchical position accommodate their emoji usage more often $(M=.41, S E=.27)$ than participants in a high hierarchical position $(M=-.01, S E=.34)$. This difference, 0.42 , between the accommodation of emoji of participants in a low hierarchical position and in a high hierarchical position was significantly different, $t(120)=7.57, p<.001$, with an effect size of $r=0.57$.

A linear regression was conducted for testing hypothesis 2 with perceived hierarchy position as the independent variable $(M=3.66, S D=1.23)$ and emoji accommodation as the dependent variable $(M=$ $0.20, S D=0.37)$. The correlation between perceived hierarchical position and emoji accommodation was checked. The results showed a moderate positive correlation between perceived hierarchy position and emoji accommodation, $r=.325, p<.001$. Homoscedasticity was tested by checking the standardized and unstandardized residuals. Auto-correlation was tested with Durbin-Watson statistic (1.45). The multiple coefficient of determination $R^{2}$ for the overall model was .11 (adjusted $R^{2}=.10$ ). Perceived hierarchy position was able to significantly predict emoji accommodation, $F(1,120)=14.15, p<.001$. An increase of perceived hierarchy position by one unit predicted an increase in emoji accommodation by .099, $B=.099, \beta=.33, t(120)=3.76, p<.001$.

To test hypothesis 3 , we conducted two t-tests for dependent samples: 1) formal with emoji versus formal without emoji, 2) informal with emoji versus informal without emoji. We used the withinsubject data we collected by means of the four different chat records that were shown in random order. Hence, we were able to check whether subjects perceived the hierarchical position differently just based on the presence of emoji as everything else remained unchanged. The first t-test of the formal chat records showed no significant difference for the perceived hierarchy with emoji $(M=3.89, S D=1.29)$ and without emoji $(M=4.07, S D=1.38), t(121)=1.03, p=.31$. Also, the second t-test showed that perceived hierarchical position of the informal chat record with emoji $(M=2.85, S D=0.64)$ does not differ significantly from the informal chat without emoji $(M=2.90, S D=0.63), t(121)=0.63, p=.53$.

\section{Discussion}

Our aim is to investigate the relationship of perceived hierarchy and emoji accommodation behaviour. So far, computer-mediated communication and instant messaging have been related to the Communication Accommodation Theory. However, we originally study whether emoji - which is deemed as a substitute for nonverbal expressions from $\mathrm{FtF}$ communication - are also adopted to the interlocutor's usage.

Like hypothesised in $\mathrm{H} 1$, we found a significant difference and stronger emoji accommodation behaviour when participants saw the chat record with a superior. Hence, emoji usage can also be subject to an asymmetrical accommodation behaviour (Giles et al. 1991). Confirming CAT that assumes accommodation asymmetry in imbalanced hierarchical situations, we found empirical indications that this applies for emoji usage which is quite different to other communication features like text length, response time or certain words (Riordan et al. 2013; Scissors et al. 2009). Using different phrases than usual might be easier and contextual in anyway - i.e. you simply write an application or motivation letter differently than a cancellation letter. In contrast, emoji can have different meanings to different people which could make an accommodation less likely. Still, our study demonstrates that people who usually 
do not use emoji (calculations from the baseline) would use them if the interlocutor is perceived as superior.

Besides the average difference, we also tested a linear effect of perceived hierarchical position. The regression model for hypothesis $\mathrm{H} 2$ showed a significance prediction of the accommodation behaviour. The higher participants perceived their interlocutors' hierarchical position, the more converging accommodation has been shown. Convergence means that participants have either positively deviated from their usual emoji baseline or reduced the number of emoji to fit the interlocutor's style. Emoji are presumably seen as an important aspect in the communication that is worth to accommodate.

Continuing on the background, nonverbal cues are of particular importance in FtF conversations. On the one hand, they can enrich the spoken word and underline the meaning and importance of the message (Wharton 2009). On the other hand, nonverbal cues can change messages' meaning and give them a different touch. Emoji can fill the gap in computer-mediated communication and provide a means to convey nonverbal cues that are not less important in such situations. Before the introduction and diffusion of emoji, people may have learned that digital text messages (e.g. e-mails) should be treated carefully as a receiver may read a message differently than the sender meant. In particular, organisations may face such situations as more and more FtF meetings are being replaced by emails. Our findings underline the importance of emojis as nonverbal cue in instant messaging and potentially more computer-mediated communication channels. Emoji can help to underline or clarify a message's meaning (Pohl et al. 2017) which would reduce misunderstandings and ineffective communication.

The assumed positive effect on hierarchy (hypothesis 3) like found for ESN (Riemer et al. 2015) could not be confirmed. Neither the formal chats with and without emoji differed significantly nor the informal ones. However, the means are slightly different and support a general tendency. Future studies that focus on this aspect may yield more information when and why emoji usage influences the perceived hierarchical position of a conversation partner. However, this finding could also be interpreted in that emoticons or emoji do make an conversation partner appear less credible (Glikson et al. 2017). Nevertheless, we cannot answer yet whether it is advisable to appear a bit less credible but also to reduce social distance and, in turn, increase efficient collaboration, or to maintain hierarchical differences.

\section{Conclusion}

In this study, we examined to what extent the Communication Accommodation Theory (CAT) can be applied to instant messaging in situations with hierarchical imbalance. We focused on the convergence aspect of CAT and further, how emoji are accommodated in consideration of hierarchy position. In our analyses, the perceived hierarchy position has an influence on the emoji accommodation. The higher the perceived hierarchical position of an interlocutor, the more emoji usage is converged. Individuals that normally enjoy the usage of many emoji in their text messages stopped using the high amount and started to use less emoji when texting with a person in a higher hierarchical position. Individuals that use almost no emoji started to increase their emoji frequency when texting with an individual in a higher hierarchical position. Hence, this study is able to extend CAT by a further aspect - emoji usage in instant messaging.

Besides such theoretical contributions, our study can help companies and practitioners to understand the effect of hierarchy on conversations and, in particular, on emoji usage. When emoji are suggested as a means to diminish misunderstandings in textual ad-hoc conversations, our study indicates that superiors need to introduce emoji because subordinates accommodate the behaviour. Furthermore, our study showed no significant influence of emoji on the perceived hierarchy which has been assumed in prior works. Since language is alive and constantly changing, using emoji in a business environment can be worth an attempt to improve communication.

However, our study is not without limitations. Participants had to select from a set of emoji to add them to a message. Participants could pick from 15 frequently used emoji only. It could have happened that they did not pick any emoji because their favourites were not available. Furthermore, the emoji were presented next to check boxes which the participants had to click when they wanted to add a certain emoji to a message. They are not used to pick emoji by activating check boxes but by touching them on a virtual keyboard. The given results should, therefore, be validated in a future research setting where participants have the possibility to formulate their answers with a familiar keyboard on their own.

Furthermore, we focused on the converging accommodation towards interlocutors in higher positions. Therefore, we designed the study to only differentiate between higher position and equal position (including the possibility of lower position). Future studies may investigate how diverging behaviour is 
influenced by the hierarchical position, potentially calling for the inclusion of a lower hierarchical position. Last, the baseline quotient was calculated based on the four chat records which can only serve as a proxy for their actual emoji usage behaviour. To address the baseline problem, it might be a solution to collect participants' chat records of real conversations with e.g. a superior, a friend or a relative. With the help of the chat records and the used emoji during the conversations, a baseline could be calculated which better reflects the real usage of emoji.

\section{References}

An, J., Li, T., Teng, Y., and Zhang, P. 2018. "Factors Influencing Emoji Usage in Smartphone Mediated Communications," Springer International Publishing, pp. 423-428 (doi: 10.1007/978-3-31978105-1).

Bisel, R. S., Messersmith, A. S., and Kelley, K. M. 2012. "Supervisor-Subordinate Communication: Hierarchical Mum Effect Meets Organizational Learning," Journal of Business Communication (49:2), pp. 128-147 (doi: 10.1177/0021943612436972).

Bunz, U., and Campbell, S. W. 2004. "Politeness Accommodation in Electronic Mail," Communication Research Reports (21:1), pp. 11-25 (doi: 10.1080/08824090409359963).

Chen, Z., Lu, X., Shen, S., Ai, W., Liu, X., and Mei, Q. 2017. “Through a Gender Lens: An Empirical Study of Emoji Usage over Large-Scale Android Users," in Human-Computer Interaction, pp. 1-20 (doi: 10.1145/3178876.3186157).

Cheung, C. M. K., Shen, A. X. L., Lee, M. K. O., and Wang, W. P. 2007. "Let`s Work Together! WeIntention to Use Instant Messaging for E-Collaboration," European Conference on Information Systems (ECIS) (2007), pp. 407-418.

Church, K., and de Oliveira, R. 2013. "What's up with WhatsApp? Comparing Mobile Instant Messaging Behaviors with Traditional SMS," in 15th International Conference on Human-Computer Interaction with Mobile Devices and Services (MobileHCI'13), pp. 352-361 (doi: 10.1145/2493190.2493225).

Dragojevic, M., Gasiorek, J., and Giles, H. 2016. “Accommodative Strategies as Core of the Theory,” in Communication Accommodation Theory: Negotiating Personal and Social Identities Across ContextsH. Giles (ed.), University of California, Santa Barbara: Cambridge University Press, pp. 36-59 (doi: 10.1017/CBO9781316226537.003).

Elder, A. M. 2018. "What Words Can't Say Emoji and Other Non-Verbal Elements of TechnologicallyMediated Communication," Journal of Information, Communication and Ethics in Society (16:1), pp. 2-15 (doi: 10.1108/JICES-08-2017-0050).

Giles, H., Coupland, N., and Coupland, J. 1991. "Accomodation Theory: Communication, context, and consequence," Contexts of Accomodation: Developments in Applied Cociolinguistics, pp. 1-68 (doi: 10.1017/CBO9780511663673.001).

Giles, H., and Ogay, T. 2007. “Communication Accommodation Theory," in Explaining communication: Contemporary theories and exemplarsB. B. Whaley and W. Samter (eds.), Mahwah, NJ: Lawrence Erlbaum, pp. 293-310 (doi: 10.1007/978-1-349-25582-5_19).

Gilson, L. L., Maynard, M. T., Jones Young, N. C., Vartiainen, M., and Hakonen, M. 2015. "Virtual Teams Research: 10 Years, 10 Themes, and 10 Opportunities," Journal of Management (41:5), pp. 13131337 (doi: 10.1177/0149206314559946).

Glikson, E., Cheshin, A., and van Kleef, G. A. 2017. "The Dark Side of a Smiley: Effects of Smiling Emoticons on Virtual First Impressions," Social Psychological and Personality Science, pp. 1-12 (doi: 10.1177/1948550617720269).

Halevy, N., Y. Chou, E., and D. Galinsky, A. 2011. "A functional model of hierarchy: Why, how, and when Vertical Differentiation Enhances Group Performance,” Organizational Psychology Review (1:1), pp. 32-52 (doi: 10.1177/2041386610380991).

Jaeger, S. R., Xia, Y., Lee, P. Y., Hunter, D. C., Beresford, M. K., and Ares, G. 2018. "Emoji Questionnaires Can Be Used with a Range of Population Segments: Findings Relating to Age, Gender and Frequency of Emoji/Emoticon Use," Food Quality and Preference (68), Elsevier, pp. 397-410 (doi: 10.1016/j.foodqual.2017.12.011). 
Jones, E., Gallois, C., Callan, V., and Barker, M. 1999. "Strategies of Accommodation: Development of a Coding System for Conversational Interaction," Journal of Language and Social Psychology (18:2), pp. 123-151 (doi: 10.1177/0261927X99018002001).

Jones, S., Cotterill, R., Dewdney, N., Muir, K., and Joinson, A. 2014. "Finding Zelig in Text: A Measure for Normalising Linguistic Accommodation," in COLING 2014, the 25th International Conference on Computational Linguistics, pp. 455-465.

Kirkman, B. L., Rosen, B., Gibson, C. B., Tesluk, P. E., and McPherson, S. O. 2002. "Five challenges to virtual team success: Lessons from Sabre, Inc.," Academy of Management Executive (16:3), pp. 67-79 (doi: 10.5465/AME.2002.8540322).

Koski, J. E., Xie, H., and Olson, I. R. 2015. "Understanding Social Hierarchies: The Neural and Psychological Foundations of Status Perception," Social Neuroscience (10:5), pp. 527-550 (doi: 10.1080/17470919.2015.1013223).

Lee, Y. K., Chang, C. T., Lin, Y., and Cheng, Z. H. 2014. "The Dark Side of Smartphone Usage: Psychological Traits, Compulsive Behavior and Technostress," Computers in Human Behavior (31), Elsevier Ltd, pp. 373-383 (doi: 10.1016/j.chb.2013.10.047).

Li, W., Chen, Y., Hu, T., and Luo, J. 2018. "Mining the Relationship between Emoji Usage Patterns and Personality," in The International AAAI Conference on Web and Social Media (ICWSM).

Lohmann, K., Pyka, S. S., and Zanger, C. 2017. “The Effects of Smileys on Receivers' Emotions,” Journal of Consumer Marketing (34:6), pp. 489-495 (doi: 10.1108/JCM-02-2017-2120).

Lu, X., Ai, W., Liu, X., Li, Q., Wang, N., Huang, G., and Mei, Q. 2016. "Learning from the Ubiquitous Language: an Empirical Analysis of Emoji Usage of Smartphone Users," in ACM International Joint Conference on Pervasive and Ubiquitous Computing - UbiComp '16, pp. 770-780 (doi: 10.1145/2971648.2971724).

Ludwig, S., de Ruyter, K., Mahr, D., Wetzels, M., and Bruggen, E. 2014. "Take Their Word for It: the Symbolic Role of Linguistic Style Matches in User Communities," MIS Quarterly Quarterly (38:4), pp. 1201-1217 (doi: 10.25300/MISQ/2014/38.4.12).

Meske, C., Kissmer, T., and Stieglitz, S. 2018. "Global adoption of unified communication technologies as part of digital transformation in organizations: A cross-cultural perspective," Multikonferenz Wirtschaftsinformatik (MKWI), pp. 133-144 (doi: 10.1021/jpo600817).

Mirbabaie, M., Stieglitz, S., and Ruiz Eiro, M. 2017. "\#IronyOff: Understanding the usage of irony on Twitter during a corporate crisis.," Pacific Asia Conference on Information Systems (PACIS).

Muir, K., Joinson, A., and Cotterill, R. 2016. "When Communication Accommodation Backfires: Interpersonal Effects of Social Power and Linguistic Style Accommodation in Computer-MediatedCommunication," in 66th International Communication Association Annual Conference.

Muir, K., Joinson, A., Cotterill, R., and Dewdney, N. 2016. "Characterizing the Linguistic Chameleon: Personal and Social Correlates of Linguistic Style Accommodation," Human Communication Research (42:3), pp. 462-484 (doi: 10.1111/hcre.12083).

Muir, K., Joinson, A., Cotterill, R., and Dewdney, N. 2017. "Linguistic Style Accommodation Shapes Impression Formation and Rapport in Computer-Mediated Communication," Journal of Language and Social Psychology (36:5), pp. 525-548 (doi: 10.1177/0261927X17701327).

Myers, K. K. 2016. "Supervisor - Subordinate Communication," The International Encyclopedia of Interpersonal Communication, pp. 1-9 (doi: 10.1002/9781118540190.wbeico045).

Park, S., Cho, K., and Lee, B. G. 2014. "What Makes Smartphone Users Satisfied with the Mobile Instant Messenger ?: Social Presence, Flow, and Self-disclosure," International Journal of Multimedia and Ubiquitous Engineering (9:11), pp. 315-324 (doi: 10.14257/ijmue.2014.9.11.31).

Pohl, H., Domin, C., and Rohs, M. 2017. "Beyond Just Text: Semantic Emoji Similarity Modeling to Support Expressive Communication," ACM Transactions on Computer-Human Interaction (24:1), pp. 1-42 (doi: 10.1145/3039685).

Quan-Haase, A., Cothrel, J., and Wellman, B. 2005. "Instant Messaging for Collaboration: A Case Study of a High-Tech Firm," Journal of Computer-Mediated Communication (10:4) (doi: 10.1111/j.10836101.2005.tbo0276.x). 
Richmond, V. P., and McCroskey, J. C. 200o. "The Impact of Supervisor and Subordinate Immediacy on Relational and Organizational Outcomes," Communication Monographs (67:1), pp. 85-95 (doi: 10.1080/03637750009376496).

Riemer, K., Stieglitz, S., and Meske, C. 2015. "From Top to Bottom: Investigating the Changing Role of Hierarchy in Enterprise Social Networks," Business and Information Systems Engineering (57:3), pp. 197-212 (doi: 10.1007/s12599-015-0375-3).

Riordan, M. A. 2017a. "Emojis as Tools for Emotion Work: Communicating Affect in Text Messages," Journal of Language and Social Psychology (36:5), pp. 549-567 (doi: 10.1177/0261927X17704238).

Riordan, M. A. 2017b. "The Communicative Role of Non-Face Emojis: Affect and Disambiguation," Computers in Human Behavior (76), Elsevier Ltd, pp. 75-86 (doi: 10.1016/j.chb.2017.07.009).

Riordan, M. A., Markman, K. M., and Stewart, C. O. 2013. "Communication Accommodation in Instant Messaging: An Examination of Temporal Convergence," Journal of Language and Social Psychology (32:1), pp. 84-95 (doi: 10.1177/0261927X12462695).

Scissors, L. E., Gill, A. J., Geraghty, K., and Gergle, D. 2009. "In CMC We Trust: The Role of Similarity," in 27th International Conference on Human Factors in Computing Systems - CHI o9, pp. 527536 (doi: 10.1145/1518701.1518783).

Skovholt, K., Grønning, A., and Kankaanranta, A. 2014. "The Communicative Functions of Emoticons in Workplace E-Mails: :-)," Journal of Computer-Mediated Communication (19:4), pp. 780-797 (doi: 10.1111/jec4.12063).

Stieglitz, S., Brockmann, T., and Mirbabaie, M. 2014. "How Context Impacts on Media Choice," 25th Australasian Conference on Information Systems (ACIS).

Tandyonomanu, D., and Tsuroyya. 2018. "Emoji: Representations of Nonverbal Symbols in Communication Technology," in IOP Conference Series: Materials Science and Engineering (Vol. 288) (doi: 10.1088/1757-899X/288/1/012052).

Tigwell, G. W., and Flatla, D. R. 2016. ““Oh that's what you meant!”: Reducing Emoji Misunderstanding," in 18th International Conference on Human-Computer Interaction with Mobile Devices and Services Adjunct (MobileHCI '16), pp. 859-866 (doi: 2957265.2961844).

Waldeck, J. H., Kearney, P., and Plax, T. G. 2012. Business and Professional Communication in a Digital Age, Cengage Learning.

Walther, J. B., and D'Addario, K. P. 2001. "The Impacts of Emoticons on Message Interpretation in Computer-Mediated Communication," Social Science Computer Review (19:3), pp. 324-347.

Wang, J., and Gallivan, M. 2009. "An Empirical Study on the Adoption of Instant Messages for Work Purposes An Empirical Study on the Adoption of Instant Messengers for Work Purposes," Information Systems.

Wharton, T. 2009. "Natural pragmatics," in Pragmatics and Non-Verbal Communication, pp. 1-17 (doi: 10.1017/CBO9780511635649).

Wijeratne, S., Balasuriya, L., Sheth, A., and Doran, D. 2017. "A Semantics-Based Measure of Emoji Similarity," in International Conference on Web Intelligence, pp. 646-653 (doi: 10.1145/3106426.3106490).

Acknowledgement: Tobias Kroll has received a funded PhD scholarship from the Foundation of German Business.

Copyright: (C) 2018 Tobias Kroll, Lea-Marie Braun, and Stefan Stieglitz. This is an open-access article distributed under the terms of the Creative Commons Attribution-NonCommercial 3.0 Australia License, which permits non-commercial use, distribution, and reproduction in any medium, provided the original author and ACIS are credited. 